\title{
NMR-based metabonomic analysis of the hepatotoxicity induced by combined exposure to PCBs and TCDD in rats
}

\author{
Chunfeng Lu ${ }^{\text {a,b,1 }}$, Yimei Wang ${ }^{\mathrm{a}, 1}$, Zhiguo Sheng ${ }^{\mathrm{c}}$, Gang Liu ${ }^{\mathrm{a}}, \mathrm{Ze} \mathrm{Fu}^{\mathrm{a}}$, Jing Zhao ${ }^{\mathrm{a}}$, Jun Zhao ${ }^{\mathrm{a}}$, Xianzhong Yan ${ }^{\mathrm{d}}$, \\ Benzhan Zhu ${ }^{c}$, Shuangqing Peng a,*

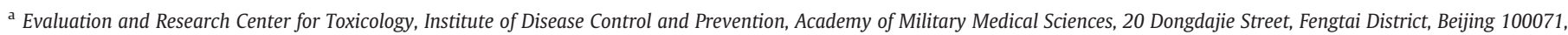 \\ PR China \\ ${ }^{\mathrm{b}}$ Department of Pharmacology, Basic Medical College, Jiamusi University, Jiamusi 154007, PR China \\ ' State Key Laboratory of Environmental Chemistry and Ecotoxicology, Research Center for Eco-Environmental Sciences, Chinese Academy of Sciences, Beijing 100085, \\ People's Republic of China \\ d National Center of Biomedical Analysis, 27 Taiping Road, Beijing 100850, PR China
}

\section{A R T I C L E I N F O}

\section{Article history:}

Received 25 March 2010

Revised 6 July 2010

Accepted 23 July 2010

Available online 5 August 2010

\section{Keywords:}

Polychlorinated biphenyls

2,3,7,8-Tetrachlorodibenzo-p-dioxin

Combined exposure

Hepatotoxicity

NMR

Metabonomics

\begin{abstract}
A B S T R A C T
A metabonomic approach using ${ }^{1} \mathrm{H}$ NMR spectroscopy was adopted to investigate the metabonomic pattern of rat urine after oral administration of environmental endocrine disruptors (EDs) polychlorinated biphenyls (PCBs) and 2,3,7,8-tetrachlorodibenzo- $p$-dioxin (TCDD) alone or in combination and to explore the possible hepatotoxic mechanisms of combined exposure to PCBs and TCDD. ${ }^{1} \mathrm{H}$ NMR spectra of urines collected $24 \mathrm{~h}$ before and after exposure were analyzed via pattern recognition by using principal component analysis (PCA). Serum biochemistry and liver histopathology indicated significant hepatotoxicity in the rats of the combined group. The PCA scores plots of urinary ${ }^{1} \mathrm{H}$ NMR data showed that all the treatment groups could be easily distinguished from the control group, so could the PCBs or TCDD group and the combined group. The loadings plots of the PCA revealed remarkable increases in the levels of lactate, glucose, taurine, creatine, and 2-hydroxy-isovaleric acid and reductions in the levels of 2-oxoglutarate, citrate, succinate, hippurate, and trimethylamine- $\mathrm{N}$-oxide in rat urine after exposure. These changes were more striking in the combined group. The changed metabolites may be considered possible biomarker for the hepatotoxicity. The present study demonstrates that combined exposure to PCBs and TCDD induced significant hepatotoxicity in rats, and mitochondrial dysfunction and fatty acid metabolism perturbations might contribute to the hepatotoxicity. There was good conformity between changes in the urine metabonomic pattern and those in serum biochemistry and liver histopathology. These results showed that the NMR-based metabonomic approach may provide a promising technique for the evaluation of the combined toxicity of EDs.
\end{abstract}

(c) 2010 Elsevier Inc. All rights reserved.

\section{Introduction}

Endocrine disruptors (EDs), as widely dispersed environmental and food contaminants that pose a great risk to public health, have been one of the widespread concerns in health safety, and of especial concern is their toxicities of combined exposure. Polychlorinated biphenyls (PCBs) and 2,3,7,8-tetrachlorodibenzo-p-dioxin (TCDD) are typical EDs and persistent organic pollutants (POPs). They are currently one of the hot spots in the field of environmental toxicology because of their extensive disposition and coexistence in the environment, persistence, bioaccumulation, migration, and high toxicity (Walker et al., 2005; Nagayama et al., 2007; Aragon et al., 2008; Ma, 2009). Most researches on the toxicity and human health hazards of these EDs have focused on toxicological studies on

\footnotetext{
* Corresponding author. Fax: + 861066948462.

E-mail address: pengsq@hotmail.com (S. Peng).

1 These authors contributed equally to this work.
}

individual compounds so that conventional techniques for toxicity evaluation remain the mainstream while the possible interactions of combined exposure to EDs have rarely been investigated systematically (Wade et al., 2002; Desaulniers et al., 2005). Considering the fact that humans are ubiquitously simultaneously exposed daily to complex mixtures of environmental contaminants, the assessment of the mixture effects of multiple pollutants using a new approach is of more theoretical and actual significance.

Metabonomics, the global analysis of metabolites, is defined as the "quantitative measurement of time-related multiparametric metabolic response of living systems to pathophysiological stimuli or genetic modification" (Nicholson et al., 1999). More practically, metabonomics is achieved by maximum data captured from biofluid or tissue analysis through the use of techniques such as nuclear magnetic resonance (NMR) spectroscopy or mass spectrometry, followed by pattern recognition statistics (Tiziani et al., 2009). This technique represents a potentially powerful method for determining the systemic response to toxicity or disease. NMR spectroscopy is one 
of the major techniques used in metabonomic studies. NMR spectra possess a wealth of metabolic information, containing signals from thousands of individual metabolites which are observed simultaneously and which can partially overlap. Because of the information density of NMR spectra, data are routinely analyzed by a combination of data reduction and pattern recognition methods using multivariate statistical analysis, such as principal components analysis (PCA) (Holmes and Antti, 2002). This strategy results in the identification of groups with similar metabolic patterns. In addition, individual metabolites discriminating between these experimental groups can be identified. The PCA is a standard technique for pattern recognition and multivariate data analysis, particularly suited to the analysis of a set of data where each individual measurement contains a multitude of data-as is the case with a series of NMR spectra, containing signals from hundreds of individual metabolites. PCA compares all measurements with each other and explains the variation inherent in the dataset in terms of artificial components called principal components (PCs). The first principal component (PC1) is responsible for the largest amount of variation in the data; the second $\mathrm{PC}$ is orthogonal to PC1 and explains the largest amount of unexplained variation, and so forth with higher PCs. In effect, the first two PCs explain the vast majority of variation present in the data.

Over the past 10 years, increasing researches have demonstrated that metabonomic data are useful for assessment of toxic mechanisms, prediction of toxicity, and identification of clinically useful biomarkers (Jordan and Cheng, 2007; Bain et al., 2009). The most common metabolites identified by NMR analyses, especially in urine, are typically those associated with major endogenous metabolic pathways such as the tricarboxylic acid (TCA) cycle, and include intermediates (Nicholson et al., 2002). However, there is clear evidence that the pattern of changes noted, even with these commonly occurring metabolites, is altered in a toxicity- and tissue-specific manner, a feature that is extremely powerful with respect to the predictive utility of metabonomic data. At the same time, there is the potential to identify unique and/or specific endogenous metabolites that are associated with a specific type of change, enabling the identification of biomarkers of toxic changes. It was shown that urine NMR analysis had at least a 4- to 16-fold higher sensitivity than histopathology and clinical chemistry in detecting adaptive and/or toxic changes caused by some compounds (Schoonen et al., 2007). The high sensitivity of NMR urinalysis and the available knowledge from other studies which apply urine NMR spectroscopy to determine the effects of hepatotoxic compounds (Kleno et al., 2004; Azmi et al., 2005; Bollard et al., 2005) led us to the belief that the NMR approach to hepatotoxicity is feasible (DellaVecchia et al., 2007). Metabolomics has been successfully applied in the research fields of pharmacology, toxicology (Nicholson n et al., 2002; Keun, 2006), and medical screening (Odunsi et al., 2005; Serkova et al., 2007; Lewis et al., 2008). However, very few studies concerning the metabonomics of TCDD and PCBs have been reported.

In our recent study, it was proved that combined exposure to TCDD and PCBs induced hepatotoxicity in rats. However, the hepatotoxic mechanism, especially the changes in metabolic profiling, remains unknown. In the present study, ${ }^{1} \mathrm{H}$ NMR-based metabonomic approach was used to reveal the possible hepatotoxic mechanisms and the possible biomarkers of combined exposure to TCDD and PCBs. Moreover, the feasibility of studying the combined toxicities of EDs metabonomically was also evaluated.

\section{Materials and methods}

Reagents and chemicals. TCDD was purchased from Cerilliant Corporation (Round Rock, TX; Lot no. ER011005-01) and the purity was 99\%. Aroclor 1254 was obtained from AccuStandard, Inc (New Haven, CT; Lot no. 124-191-B). 2,2',3,3'-Deuterotrimethylsilylproprionic acid (TSP) was purchased from Merck Corporation. All other chemicals were of analytical grade and commercially available.
Animals and housing. Male Sprague-Dawley (SD) rats of 6-8 weeks of age (180-200 g) were purchased from Beijing Vital River Laboratory Animal Co. (Beijing, China) and bred in the Laboratory Animal Center of Academy of Military Medical Sciences (Beijing, China). The laboratory conditions were maintained under a $12 \mathrm{~h}$ light/dark cycle at $22 \pm 2{ }^{\circ} \mathrm{C}$ and $50 \pm 10 \%$ relative humidity. The animals were provided with standard commercial pelleted feed and water ad libitum.

Experimental design. All animal procedures were approved by the Institutional Animal Care and Use Committee of Academy of Military Medical Sciences and were performed in strict accordance with current animal care guidelines. Animals were allowed to acclimate for 1 week before the start of the study. After acclimatization, the animals were assigned randomly into four groups of five each, including a control group and three treatment groups. Rats in the treatment groups received $10 \mu \mathrm{g} / \mathrm{kg}$ of TCDD, $10 \mathrm{mg} / \mathrm{kg}$ of PCBs (Aroclor 1254) or the combination of TCDD $(10 \mu \mathrm{g} / \mathrm{kg})$ and Aroclor $1254(10 \mathrm{mg} / \mathrm{kg})$, respectively. Control rats were administered with equivalent volumes of olive oil only. All animals were treated by intragastric administration $(2.5 \mathrm{ml} / \mathrm{kg}$ ) daily for 6 consecutive days. TCDD and Aroclor 1254 were initially dissolved in DMSO and further diluted with olive oil.

Rats were individually housed in metabolism cages, body weights were recorded daily, and the urine samples in 24 hours were collected before and after exposure. The urine samples were kept at $-80^{\circ} \mathrm{C}$ pending ${ }^{1} \mathrm{H}$ NMR spectroscopic analyses. On the next morning of the last administration, the rats were subject to anesthesia, and the blood was collected for serum biochemical analysis. The livers were immediately isolated and weighed, and the left lobe of each liver was stored in formalin for histopathological evaluation.

Serum biochemical analysis. Biochemical analyses of the serum obtained from the collected blood were performed by an automated analyzer (Hitachi, Japan) for the following parameters: activity of alanine aminotransferase (ALT), aspartate aminotransferase (AST) and alkaline phosphatase (ALP), and the levels of cholesterol (CHOL), triglyceride (TG) and total bilirubin (TBIL). Data were presented as mean \pm standard deviations (SD). Statistical analyses were performed using the two-way analysis of variance (2-way ANOVA) followed by the post-hoc Dunnett $t$ test. A $p$ value less than 0.05 was regarded as statistically significant.

Histopathological examination. Formalin-fixed livers were processed according to the routine procedure, and $4 \mu \mathrm{m}$ of thickness was sectioned. The sections were stained with haematoxylin and eosin (H\&E), and the slides were examined by an expert in histopathologic evaluation. Histopathologic alterations were evaluated with a grading scale: NC, no obvious changes; + , minimal alterations; ++ , intermediate alterations; and +++ , extensive alterations.

${ }^{1} \mathrm{H}$ NMR spectroscopy. The preparation of the urine for NMR analysis was based on a protocol previously described (Beckwith-Hall et al., 1998). Urine samples were prepared by mixing $400 \mu$ of urine with $200 \mu$ l of $0.2 \mathrm{M}$ sodium phosphate buffer ( $\mathrm{pH} 7.4$ ) and kept for $10 \mathrm{~min}$. Then the samples were centrifuged at $13,000 \times g$ for $10 \mathrm{~min}$ to remove any precipitates. Five hundred-microliter aliquots of the resulting supernatant were transferred into 5-mm NMR tubes, to which $50 \mu \mathrm{l}$ of a solution of TSP in deuterium oxide $\left(\mathrm{D}_{2} \mathrm{O}\right)$ was added (final concentration $=1 \mathrm{mM}$ ). The $\mathrm{D}_{2} \mathrm{O}$ and TSP provided a field frequency lock for the NMR spectrometer and the chemical shift reference $(\delta 0.0)$, respectively. ${ }^{1} \mathrm{H}$ NMR spectra of all the urine samples were obtained at $599.69 \mathrm{MHz}{ }^{1} \mathrm{H}$ frequency by using a Varian INOVA 600 NMR spectrometer. One-dimensional spectrum was acquired by using a standard NOESY pulse sequence with water suppression during the mixing time of $0.15 \mathrm{~s}$ and the relaxation delay of $2 \mathrm{~s}$. Sixty-four free induction decays (FIDs) were collected into $64 \mathrm{~K}$ data points, and the 
number of scans collected for each urine sample was 256 . The data were zero-filled by a factor, and the FIDs were multiplied by an exponential function equivalent to a line broadening of $0.3 \mathrm{~Hz}$ Fourier transformation (FT).

${ }^{1}$ H NMR data analysis. All NMR spectra were phased and baseline corrected and then data-reduced to 225 integrated regions of equal width $(0.04 \mathrm{ppm})$ corresponding to the region of $\delta 0.52$ to $\delta 9.48$ using the VNMR 6.1C software package (Varian, Inc). The region of $\delta 4.6$ to $\delta 6.2$ in the urine spectra was excluded from the recognition analysis to remove the uncertainty of residual water signal and urea. Normalization to the total sum of the spectrum was carried out on the data before pattern recognition analyses, and the values of all variables were mean-centered and pareto-scaled (Webb-Robertson et al., 2005; Craig et al., 2006) before PCA using SIMCA-P 10.0 software package (Umetrics $A B$, Umeå, Sweden). Pareto scaling gives each variable a variance numerically equal to its standard deviation. Data were visualized by using the first two PCs scores and loadings plots. Each point on the scores plot represents an individual sample, and each point on the loadings plot represents a single NMR spectral region that was altered as a result of PCBs or TCDD dosing. The scores and loadings plots are complementary. Mean data were calculated for each integrated regions for each time point. Thus, biochemical components responsible for the differences between samples detected in the scores plot can be extracted from the corresponding loadings.

\section{Results}

\section{Body weight and relative liver weight}

There was no significant difference in body weights of rats in the PCBs group when compared with the control group. However, body weights in the TCDD and the combined groups were significantly reduced from the 5 th and 4 th days, respectively, as compared with the control group, and the maximal reduction was found in the combined group (Table 1 ).

The relative liver weight (liver weight / body weight $\times 100 \%$ ) in all the treatment groups showed significant increase when compared with the control group $(p<0.05)$, and the maximal increase was found in the combined group $(p<0.05)$. There was significant difference between the PCBs or TCDD group and the combined group $(p<0.05$; Table 1).

\section{Serum biochemistry}

Biochemical changes of serum are presented in Table 2. Combined exposure to PCBs and TCDD induced significant changes in serum biochemistry. Serum TG, CHOL, TBIL levels, and AST activity were increased by $85.7 \%, 163.8 \%, 104.5 \%$, and $112.6 \%$ as compared to the control value, respectively ( $p<0.05)$, indicating the occurrence of liver injuries. In addition, there were statistical differences in several biochemical parameters between the PCBs or TCDD group and the combined group $(p<0.05)$.

\section{Table 1}

Effects of exposure to PCBs and TCDD alone or in combination on body weights and relative liver weights of SD rats. Results are displayed as the mean $\pm S D$ of five animals per group. Two-way ANOVA was used followed by the post-hoc Dunnett $t$ test.

\begin{tabular}{lllcl}
\hline Treatment & $n$ & Body weight $(\mathrm{g})$ & Liver weight $(\mathrm{g})$ & Relative liver weight $(\%)$ \\
\hline Control & 5 & $227.9 \pm 14.1$ & $7.0 \pm 0.4$ & $3.1 \pm 0.2$ \\
PCBs & 5 & $221.3 \pm 10.5^{\mathrm{a}}$ & $11.3 \pm 0.4^{\mathrm{b}}$ & $5.1 \pm 0.2^{\mathrm{a}, \mathrm{b}}$ \\
TCDD & 5 & $203.0 \pm 3.9^{\mathrm{b}}$ & $9.3 \pm 1.1^{\mathrm{a}, \mathrm{b}}$ & $4.6 \pm 0.5^{\mathrm{a}, \mathrm{b}}$ \\
TCDD + PCBs & 5 & $181.7 \pm 15.1^{\mathrm{b}}$ & $12.2 \pm 1.2^{\mathrm{b}}$ & $6.7 \pm 0.5^{\mathrm{b}}$ \\
\hline
\end{tabular}

\footnotetext{
a $p<0.05$ versus combined group.
}

b $p<0.05$ versus control group.

\section{Liver histopathology}

Liver histopathologic examination was performed to further evaluate the hepatotoxicity of PCBs and TCDD. Control livers showed no signs of obvious abnormality (Fig. 1A). In contrast, there was slight hepatocellular swelling and a few vacuolations in the livers of PCBstreated rats (Fig. 1B). Mild hepatocellular swelling and vacuolation were noted in the TCDD group (Fig. 1C). Combined exposure to PCBs and TCDD induced marked swelling, mild to moderate hepatocellular vacuolation, necrosis and inflammatory infiltration in the livers (Fig. 1D). Generally, the histological changes of the liver in the combined group were more remarkable than those in the PCBs or TCDD group (Table 3).

\section{${ }^{1} \mathrm{H}$ NMR spectra of rat urine}

Typical ${ }^{1} \mathrm{H}$ NMR spectra obtained from urine samples of rats exposed to PCBs and TCDD after 1, 3, and 6 days were shown in Fig. 2. The spectra were dominated by a number of metabolites, including citrate, trimethylamine- $\mathrm{N}$-oxide (TMAO), 2-oxoglutarate, creatine, creatinine, lactate, succinate, taurine, dimethylamine (DMA), hippurate, 2-hydroxy-isovalerate, 2-oxoisovalerate and glucose. ${ }^{1} \mathrm{H}$ NMR spectra revealed differences in overall composition between the urines from control animals and PCBs and TCDD-treated animals. Most changes in the ${ }^{1} \mathrm{H}$ NMR spectra are related to endogenous metabolites involved in carbohydrate metabolism such as the TCA cycle intermediates, e.g., citrate, 2-oxoglutarate, succinate, and the glycolysis end product lactate (Fig. 2C).

\section{PCA of ${ }^{1} \mathrm{H}$ NMR data}

The PCA was performed to facilitate a general overview of metabolite patterns and to identify potential biomarkers. The PCA scores plots of the urinary ${ }^{1} \mathrm{H}$ NMR data are shown in Fig. 3A. Each data point corresponds to one NMR spectrum and represents one urine sample, and the position in the plot was determined by the difference of that spectrum with respect to the average of the respective $\mathrm{PC}$. The distance between points in the scores plot is an indication of the similarity between samples. The scores plots revealed that all the treatment groups could be easily distinguished from the control group both along PC1 and PC2, and the distinction was the most striking for the combined group. Furthermore, the PCBs or TCDD group was also separated from the combined group. The corresponding loadings plots were shown in Fig. 3A. Each data point represents one bucket (with the chemical shift indicated explicitly). The plot identifies which spectral regions are responsible for the differences between the spectra observed in the scores plots. This means that it is possible to identify from the loadings plots which spectral regions and thus which chemical compounds are responsible for any grouping of data points observed in the scores plots. Further analysis of loading factors of PCA and ${ }^{1} \mathrm{H}$ NMR spectrum itself can show biomarkers corresponding to toxicity. Compared to the control group, in the PCBs group, the major biochemical changes identified in urine from corresponding loadings plot (Fig. 3B) and ${ }^{1} \mathrm{H}$ NMR spectra were the increase in the levels of 2-hydroxy-isovaleric acid $(\delta 3.82, \delta 3.86)$, lactate $(\delta 4.14)$, and glucose $(\delta 3.62, \delta 3.66, \delta 3.74)$ and the decrease in the levels of citrate $(\delta 2.54, \delta 2.58, \delta 2.66, \delta 2.7)$, creatine $(\delta 3.02), 2$-oxoglutarate $(\delta 2.46)$, and TMAO $(\delta 3.26)(p<0.05)$. Fig. $3 C$ shows that elevation in the levels of lactate $(\delta 4.14), 2$-hydroxy-isovaleric acid $(\delta 3.82, \delta 3.86)$, and glucose $(\delta 3.74)$ was observed, while reduction in the levels of citrate $(\delta 2.54, \delta 2.58, \delta 2.66, \delta 2.7)(p<0.05)$ was found in the TCDD group. The predominant changes identified in the PCA of the urinary ${ }^{1} \mathrm{H}$ NMR of the combined group (Fig. 3D) included remarkable increase in the levels of lactate $(\delta 4.14)$, taurine $(\delta 3.42)$, creatine $(\delta 3.9), 2$-hydroxy-isovaleric acid $(\delta 3.82, \delta 3.86)$, and glucose $(\delta 3.62, \delta 3.66, \delta 3.7, \delta 3.74)$, accompanied by reduction in the levels of 2-oxoglutarate $(\delta 2.46)$, citrate $(\delta 2.54, \delta 2.58$, 
Table 2

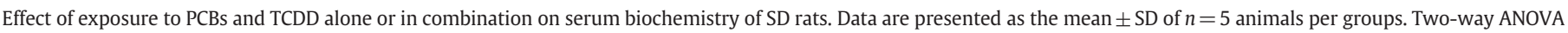
was used followed by the post-hoc Dunnett $t$ test.

\begin{tabular}{|c|c|c|c|c|c|c|c|}
\hline Treatment & $n$ & $\operatorname{ALT}(\mathrm{U} / \mathrm{L})$ & AST (U/L) & $\operatorname{ALP}(\mathrm{U} / \mathrm{L})$ & TG $(\mathrm{nmol} / \mathrm{L})$ & $\mathrm{CHOL}(\mathrm{mmol} / \mathrm{L})$ & TBIL $(\mu \mathrm{mol} / \mathrm{L})$ \\
\hline Control & 5 & $36.2 \pm 5.3$ & $178.8 \pm 23.6$ & $257.4 \pm 39.4$ & $0.35 \pm 0.06$ & $0.94 \pm 0.11$ & $0.22 \pm 0.01$ \\
\hline PCBs & 5 & $56.6 \pm 12.1$ & $205.8 \pm 51.3^{a}$ & $222.0 \pm 22.0^{\mathrm{a}}$ & $0.47 \pm 0.18$ & $1.60 \pm 0.37^{\mathrm{a}, \mathrm{b}}$ & $0.34 \pm 0.14$ \\
\hline TCDD & 5 & $35.0 \pm 4.2$ & $234.4 \pm 20.6$ & $197.2 \pm 32.7^{\mathrm{a}, \mathrm{b}}$ & $0.33 \pm 0.04^{\mathrm{a}}$ & $1.28 \pm 0.19^{a}$ & $0.52 \pm 0.22^{b}$ \\
\hline $\mathrm{TCDD}+\mathrm{PCBs}$ & 5 & $53.4 \pm 33.3$ & $380.2 \pm 59.1^{b}$ & $284.4 \pm 63.7$ & $0.65 \pm 0.36^{b}$ & $2.48 \pm 0.81^{\mathrm{b}}$ & $0.45 \pm 0.07^{b}$ \\
\hline
\end{tabular}

Abbreviations: ALT alanine aminotransferase; AST, aspartate aminotransferase; ALP, alkaline phosphatase; TG, triglyceride; CHOL, cholesterol; TBIL, total bilirubin.

${ }^{a} p<0.05$ versus combined group.

b $p<0.05$ versus control group.

$\delta 2.66, \delta 2.7)$, hippurate $(\delta 3.98, \delta 7.82)$, and succinate $(\delta 2.42)(p<0.05)$ (Table 4).

\section{Discussion}

Several studies have demonstrated that exposure to PCBs or TCDD induced hepatotoxicity (Boverhof et al., 2006; Korenaga et al., 2007). In the present research, traditional toxicity evaluations showed that all treatments elicited hepatotoxicity, as characterized by the increased liver weight, elevated serum TG and CHOL levels and AST activity, and liver histopathologic alterations. Moreover, the combined exposure induced more severe hepatotoxicity than exposure to PCBs or TCDD alone. Hepatocellular vacuolation, necrosis, and inflammatory cell infiltration were observed in the histopathologic examination of the combined group.

To explore the hepatotoxic mechanism of PCBs and TCDD, the high sensitivity of NMR urinalysis technique was used. It can inform on the state and severity of target organ dysfunction because the biochemical compositions of a biofluid will be modified if cell function varies (Lindon et al., 2004). Recent studies have shown that NMR-based metabonomic approach can be used as a rapid analytical tool for the study of the effects of hepatotoxic compounds (Kleno et al., 2004; Azmi et al., 2005; Bollard et al., 2005). In this investigation, ${ }^{1} \mathrm{H}$ NMRbased metabonomic technique was utilized to study the alterations of urine metabolite profiles in rats after combined exposure to TCDD and PCBs, and the data were analyzed by using PCA. The PCA showed that the samples were primarily distributed in four regions of the scoring oval (95\% confidence intervals). The urine metabonomic patterns of each treatment group could obviously be distinguished from that of control group, so could the PCBs or TCDD group and the combined group, suggesting that all the groups had distinct metabonomic spectra. The results suggest that this metabonomic technique can be used to characterize the metabolic consequence of combined exposure to PCBs and TCDD and to elucidate the toxic effects. Conventional toxicity evaluation techniques showed that all treatments elicited different degrees of hepatotoxicity, and the combined hepatotoxicity was more severe than that of individual exposure. These results indicated that the results of urine metabonomic pattern changes were in accordance with those of conventional toxicity evaluations.

The PCA of urinary ${ }^{1} \mathrm{H}$ NMR data showed that the levels of 2oxoglutarate, citrate, succinate, creatine, taurine, lactate, TMAO,

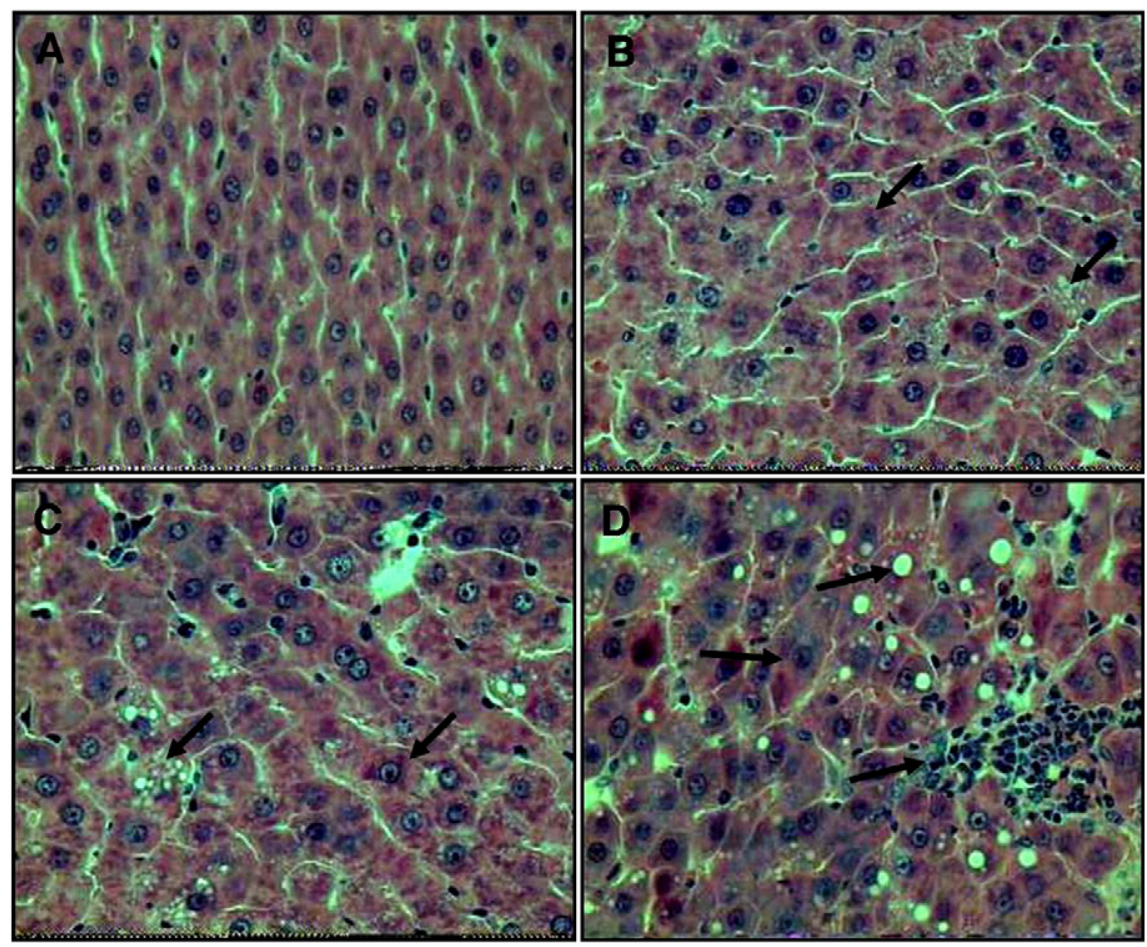

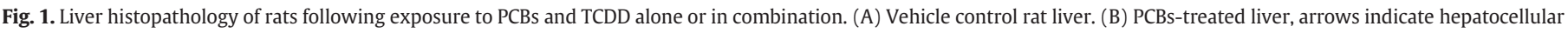

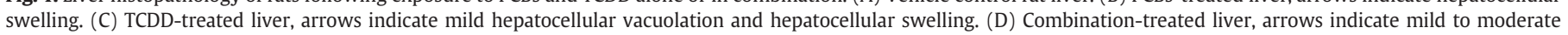
hepatocellular vacuolation and inflammatory cell infiltration. H\&E original stain, $20 \times$ objective. 
Table 3

Histopathologic alterations of rats following exposure to PCBs and TCDD alone or in combination. NC, no obvious changes; + , minimal alterations; ++ , intermediate alterations, and +++ , extensive alterations.

\begin{tabular}{llll}
\hline Treatment & $\begin{array}{l}\text { Hepatocellular } \\
\text { swelling }\end{array}$ & $\begin{array}{l}\text { Inflammatory cell } \\
\text { infiltration }\end{array}$ & $\begin{array}{l}\text { Hepatocellular } \\
\text { vacuolation }\end{array}$ \\
\hline Control & $\mathrm{NC}$ & $\mathrm{NC}$ & $\mathrm{NC}$ \\
PCBs & + & $\mathrm{NC}$ & + \\
TCDD & ++ & + & ++ \\
TCDD + PCBs & +++ & ++ & +++ \\
\hline
\end{tabular}

hippurate, 2-hydroxy-isovaleric acid, and glucose in rat urine were altered by exposure to PCBs and TCDD, and most of these changes were more evident in the combined group. These changed metabolites may be considered possible biomarkers for the combined hepatotoxicity. Furthermore, latent toxic mechanisms were hinted by analyzing the formation and the transformation of these metabolites. A prominent finding was the depletion of the TCA cycle intermediates which were mainly located in liver mitochondria, including citrate, succinate, and 2oxoglutarate, in urine samples from the combined group. These organic acids may be more universal markers for cellular liver pathology (Ishihara et al., 2006). The decreased levels of citrate, 2-oxoglutarate, and succinate in urine following PCBs and TCDD treatment could be a result of decreased fatty acid catabolism, since these metabolites are intermediates of the TCA cycle which occurs largely in liver mitochondria. When hepatic insufficiency occurs, glycolysis and gluconeogenesis are often accelerated. Enhanced gluconeogenesis and glycolysis prevent acetyl-CoA entering the TCA cycle, reducing the levels of citrate, 2-oxoglutarate, and succinate (Yokoyama et al., 2005). Similar changes were observed in ANIT, carbon tetrachloride, and hydrazineinduced hepatotoxicity (Wu et al., 2005). The reduced organic acid levels suggested that oxidative phosphorylation was inhibited, glycolysis was increased (Siculella et al., 2002; Connor et al., 2004), and that exposure to PCBs and TCDD affected the mitochondrial energy metabolism in liver, although the effect of other factors could not be excluded. The level of glucose in the urine was increased significantly in all treatment groups, which suggested that the rates of gluconeogenesis and glycolysis increased because the lipid metabolism in these animals failed. Clinical chemistry and liver histopathology examination also showed clear changes for the liver tissues, the increases of serum TG,
CHOL levels, and the appearance of vacuolation in the liver could indicate changes in fatty acid metabolism. Meanwhile, the lactate level in the urine was increased too, which offers a support to the increased rate of glycolysis. In addition, the increased levels of glucose and lactate in the urine from treated animals are consistent with mitochondrial impairment; this proposed inability may result from a lack of acetyl$\mathrm{CoA}$ in the mitochondria as a result of a reduction in the rate of oxidative phosphorylation and fatty acid $\beta$-oxidation (McGarry and Foster, 1980). These results provided evidence for the assumption that the cells switched to gluconeogenesis and glycolysis to compensate for the loss of ATP coming from fatty acid $\beta$-oxidation (Marty-Teysset et al., 1996). Our clinical chemistry data and liver histopathology examination could support it also. These indicated that mitochondrial dysfunction and abnormality of fatty acid metabolism probably were the most important mechanisms for the hepatotoxicity of PCBs and TCDD. The work of Mortishire-Smith et al. (2004) also provided evidence of altered fatty acid metabolism as a mechanism of hepatotoxicity. Wang et al. (2006) studied the hepatotoxicity of Z24 on Wistar rats by using metabonomic analysis of urinary ${ }^{1} \mathrm{H}$ NMR spectra, the results discovered that Z24 inhibited mitochondrial function through altering the energy and lipid metabolism in rats. In addition, a decrease in the formation of hippurate is another common marker for cellular liver pathology (Connor et al., 2004). Decreased oxidative phosphorylation will also lead to a decreased formation of hippurate in rat liver mitochondria. The hippurate formation depends on the metabolism of benzoate (Yavuz et al., 2005); the whole process depends on a good supply of ATP via the oxidative phosphorylation (Sprague et al., 2004). Therefore, the reduced production of ATP may lead to a reduced formation of hippurate in the combined group as observed in the present study. In addition, the levels of taurine and creatine in the urine were increased significantly in the combined group, and the combined elevations in the levels of taurine and creatine had been found previously as a biomarker for liver damage (Waterfield et al., 1993; Beckwith-Hall et al., 1998).

In summary, the present investigation has demonstrated that combined exposure to PCBs and TCDD induced significant hepatotoxicity in rats, and mitochondrial dysfunction and fatty acid metabolism perturbation might contribute to the hepatotoxicity. The metabolomic data obtained in this study provide important mechanistic clues about the effects of PCBs and TCDD on liver metabolism and energy utilization which may be important for the understanding of the pathogenesis of liver injury. We propose that the changed metabolites
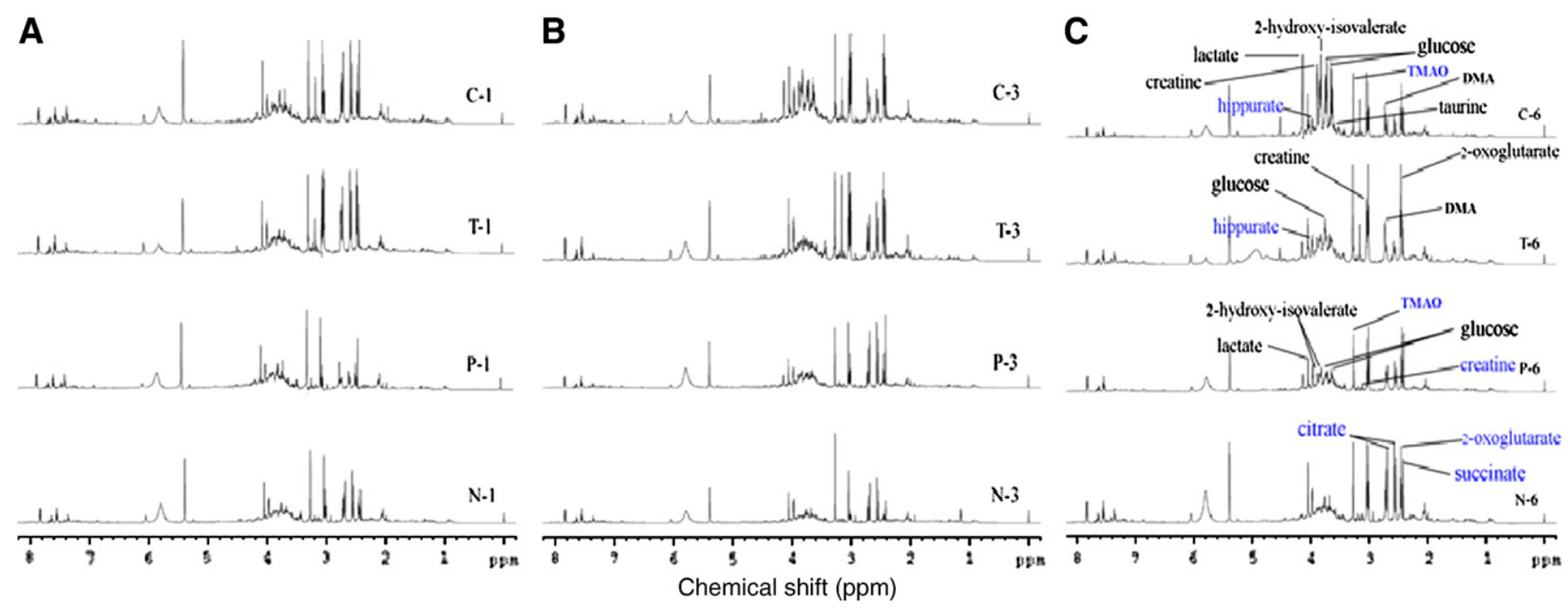

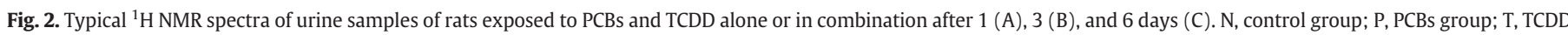
group; C, combined group. 

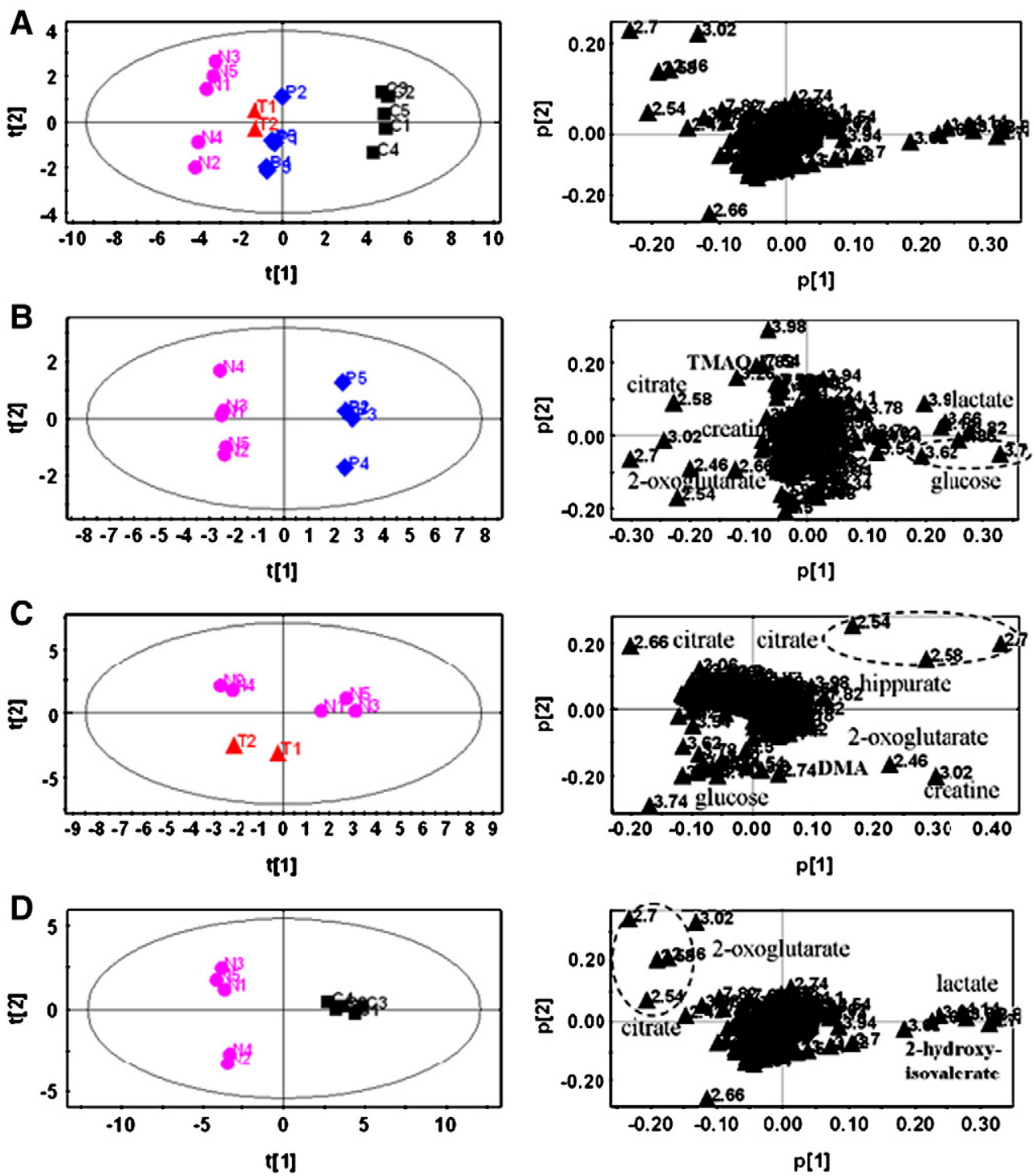

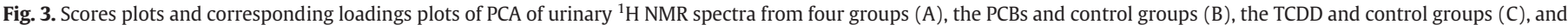

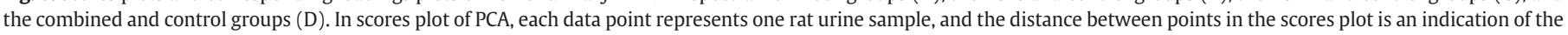

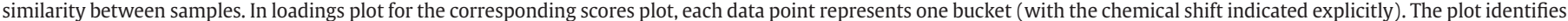

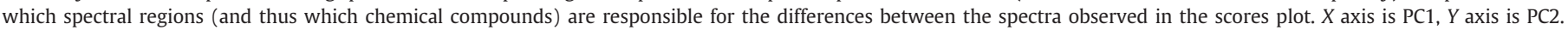
$\mathrm{N}$, control group; $\triangle \mathrm{P}, \mathrm{PCBs}$ group; $\triangle \mathrm{T}$, TCDD group; $\mathbf{} \mathrm{C}$, combined group.

could be considered as potential noninvasive biomarkers for PCBs and TCDD hepatotoxicity, while further studies will be necessary to verify these metabolites as biomarkers of PCBs and TCDD hepatotoxicity. Furthermore, there was good conformity between changes in the urine metabonomic pattern and those in serum biochemistry and liver histopathology. The results of the present study imply that the NMRbased metabonomic approach represents a promising and sensitive technique for the combined toxicity of EDs.

\section{Acknowledgments}

This work was supported by National Basic Research Program of China (973 Program) (2011CB503803), National Key Project on Drug Development from the Ministry of Science and Technology of China (2009ZX09501-034), and partly financed by the State Key Laboratory of Environmental Chemistry and Ecotoxicology, Research Center for EcoEnvironmental Sciences, Chinese Academy of Sciences (no. KF2008-20).

Table 4

Effect of PCBs and TCDD on the ${ }^{1} \mathrm{H}$ NMR spectra relative integral area of metabolites. Data are presented as the mean \pm SD of five animals per group. Two-way ANOVA was used followed by the post-hoc Dunnett $t$ test.

\begin{tabular}{|c|c|c|c|c|c|c|c|c|c|c|}
\hline Treatment & $\begin{array}{l}\text { 2-Hydroxy- } \\
\text { isovalerate }\end{array}$ & Lactate & Glucose & Taurine & Creatine & Citrate & 2-Oxoglutarate & Succinate & Hippurate & TMAO \\
\hline Control & $191.7 \pm 10.9$ & $85.7 \pm 4.6$ & $231.6 \pm 28.1$ & $82.2 \pm 13.4$ & $384.1 \pm 83.2$ & $290.2 \pm 75.5$ & $314.4 \pm 51.9$ & $210.8 \pm 13.9$ & $216.0 \pm 36.7$ & $258.7 \pm 31.76$ \\
\hline PCBs & $368.9 \pm 58.0^{\mathrm{a}}$ & $211.4 \pm 50.6^{\mathrm{a}}$ & $500.2 \pm 94.4^{\mathrm{a}}$ & $79.8 \pm 11.7$ & $231.6 \pm 48.3^{a}$ & $154.9 \pm 46.8^{\mathrm{a}}$ & $210.1 \pm 76.2^{\mathrm{a}}$ & $196.5 \pm 53.8$ & $191.9 \pm 27.7$ & $212.3 \pm 17.6^{\mathrm{a}}$ \\
\hline TCDD & $276.0 \pm 24.6^{a}$ & $159.9 \pm 23.9^{a}$ & $431.3 \pm 83.7^{a}$ & $113.8 \pm 37.4$ & $430.9 \pm 56.5$ & $122.7 \pm 5.5^{\mathrm{a}}$ & $359.7 \pm 44.7$ & $201.4 \pm 14.7$ & $177.9 \pm 5.5$ & $235.6 \pm 65.4$ \\
\hline $\mathrm{TCDD}+\mathrm{PCBs}$ & $711.3 \pm 98.1^{\mathrm{a}}$ & $513.0 \pm 79.7^{a}$ & $805.2 \pm 35.7^{\mathrm{a}}$ & $146.2 \pm 23.5^{a}$ & $506.1 \pm 57.7^{a}$ & $49.3 \pm 9.3^{\mathrm{a}}$ & $121.3 \pm 51.8^{\mathrm{a}}$ & $87.1 \pm 12.5^{a}$ & $119.8 \pm 22.9^{\mathrm{a}}$ & $220.7 \pm 53.7$ \\
\hline
\end{tabular}

a $p<0.05$ versus control group. 


\section{References}

Aragon, A.C., Kopf, P.G., Campen, M.J., Huwe, J.K., Walker, M.K., 2008. In utero and lactational 2, 3, 7, 8-tetrachlorodibenzo-p-dioxin exposure: effects on fetal and adult cardiac gene expression and adult cardiac and renal morphology. Toxicol. Sci. 101, 321-330.

Azmi, J., Griffin, J.L., Shore, R.F., Holmes, E., Nicholson, J.K., 2005. Chemometric analysis of biofluids following toxicant induced hepatotoxicity: a metabonomic approach to distinguish the effects of 1-naphthylisothiocyanate from its products. Xenobiotica 35, 839-852.

Bain, J.R., Stevens, R.D., Wenner, B.R., Ilkayeva, O., Muoio, D.M., Newgard, C.B., 2009. Metabolomics applied to diabetes research: moving from information to knowledge. Diabetes 58, 2429-2443.

Beckwith-Hall, B.M., Nicholson, J.K., Nicholls, A.W., Foxall, P.J., Lindon, J.C., Connor, S.C., Abdi, M., Connelly, J., Holmes, E., 1998. Nuclear magnetic resonance spectroscopic and principal components analysis investigations into biochemical effects of three model hepatotoxins. Chem. Res. Toxicol. 11, 260-272.

Bollard, M.E., Stanley, E.G., Lindon, J.C., Nicholson, J.K., Holmes, E., 2005. NMR-based metabonomic approaches for evaluating physiological influences on biofluid composition. NMR Biomed. 18, 143-162.

Boverhof, D.R., Burgoon, L.D., Tashiro, C., Sharratt, B., Chittim, B., Harkema, J.R. Mendrick, D.L., Zacharewski, T.R., 2006. Comparative toxicogenomic analysis of the hepatotoxic effects of TCDD in Sprague Dawley rats and C57BL/ 6 mice. Toxicol. Sci. $94,398-416$.

Connor, S.C., Wu, W., Sweatman, B.C., Manini, J., Haselden, J.N., Crowther, D.J., Waterfield, C.J., 2004. Effects of feeding and body weight loss on the ${ }^{1} \mathrm{H}-\mathrm{NMR}$-based urine metabolic profiles of male Wistar Han rats: implications for biomarker discovery. Biomarkers 9, 156-179.

Craig, A., Cloarec, O., Holmes, E., Nicholson, J.K., Lindon, J.C., 2006. Scaling and normalization effects in NMR spectroscopic metabonomic data sets. Anal. Chem. 78, 2262-2267.

DellaVecchia, M.J., Merritt, W.K., Peng, Y., Kirby, T.W., DeRose, E.F., Mueller, G.A., Van Houten, B., London, R.E., 2007. NMR analysis of [methyl- ${ }^{13} \mathrm{C}$ ]methionine UvrB from Bacillus caldotenax reveals UvrB-domain 4 heterodimer formation in solution. J. Mol. Biol. 373, 282-295.

Desaulniers, D., Cooke, G.M., Leingartner, K., Soumano, K., Cole, J., Yang, J., Wade, M. Yagminas, A., 2005. Effects of postnatal exposure to a mixture of polychlorinated biphenyls, $p, p^{\prime}$-dichlorodiphenyltrichloroethane, and $p$ - $p^{\prime}$-dichlorodiphenyldichloroethene in prepubertal and adult female Sprague-Dawley rats. Int. J. Toxicol. 24, 111-127.

Holmes, E., Antti, H., 2002. Chemometric contributions to the evolution of metabonomics: mathematical solutions to characterising and interpreting complex biological NMR spectra. Analyst 127, 1549-1557.

Ishihara, K., Katsutani, N., Aoki, T., 2006. A metabonomics study of the hepatotoxicants galactosamine, methylene dianiline and clofibrate in rats. Basic Clin. Pharmacol. Toxicol. 99, 251-260.

Jordan, K.W., Cheng, L.L., 2007. NMR-based metabolomics approach to target biomarkers for human prostate cancer. Expert Rev. Proteomics 4, 389-400.

Keun, H.C., 2006. Metabonomic modeling of drug toxicity. Pharmacol. Ther. 109, 92-106.

Kleno, T.G., Kiehr, B., Baunsgaard, D., Sidelmann, U.G., 2004. Combination of 'omics' data to investigate the mechanism(s) of hydrazine-induced hepatotoxicity in rats and to identify potential biomarkers. Biomarkers 9, 116-138.

Korenaga, T., Fukusato, T. Ohta, M., Asaoka, K., Murata, N. Arima, A., Kubota, S., 2007. Long-term effects of subcutaneously injected 2,3,7,8-tetrachlorodibenzo-p-dioxin on the liver of rhesus monkeys. Chemosphere 67, S399-S404.

Lewis, G.D., Asnani, A., Gerszten, R.E., 2008. Application of metabolomics to cardiovascular biomarker and pathway discovery. J. Am. Coll. Cardiol. 52, 117-123.

Lindon, J.C., Holmes, E., Bollard, M.E., Stanley, E.G., Nicholson, J.K., 2004. Metabonomics technologies and their applications in physiological monitoring, drug safety assessment and disease diagnosis. Biomarkers 9, 1-31.

Ma, L., 2009. Endocrine disruptors in female reproductive tract development and carcinogenesis. Trends Endocrinol. Metab. 20, 357-363.
Marty-Teysset, C., Posthuma, C., Lolkema, J.S., Schmitt, P., Divies, C., Konings, W.N 1996. Proton motive force generation by citrolactic fermentation in Leuconostoc mesenteroides. J. Bacteriol. 178, 2178-2185.

McGarry, J.D., Foster, D.W., 1980. Regulation of hepatic fatty acid oxidation and ketone body production. Annu. Rev. Biochem. 49, 395-420.

Mortishire-Smith, R.J., Skiles, G.L., Lawrence, J.W., Spence, S., Nicholls, A.W., Johnson, B.A., Nicholson, J.K., 2004. Use of metabonomics to identify impaired fatty acid metabolism as the mechanism of a drug-induced toxicity. Chem. Res. Toxicol. 17, 165-173.

Nagayama, J., Tsuji, H., Iida, T., Nakagawa, R., Matsueda, T., Hirakawa, H., Yanagawa, T., Fukushige, J., Watanabe, T., 2007. Immunologic effects of perinatal exposure to dioxins, PCBs and organochlorine pesticides in Japanese infants. Chemosphere 67, 393-398.

Nicholson, J.K., Lindon, J.C., Holmes, E., 1999. 'Metabonomics': understanding the metabolic responses of living systems to pathophysiological stimuli via multivariate statistical analysis of biological NMR spectroscopic data. Xenobiotica 29, 1181-1189.

Nicholson, J.K., Connelly, J., Lindon, J.C., Holmes, E., 2002. Metabonomics: a platform for studying drug toxicity and gene function. Nat. Rev. Drug Discov. 1, 153-161.

Odunsi, K., Wollman, R.M., Ambrosone, C.B., Hutson, A., McCann, S.E., Tammela, J., Geisler, J.P., Miller, G., Sellers, T., Cliby, W., Qian, F., Keitz, B., Intengan, M., Lele, S., Alderfer, J.L., 2005. Detection of epithelial ovarian cancer using ${ }^{1} \mathrm{H}-\mathrm{NMR}$-based metabonomics. Int. J. Cancer 113, 782-788.

Schoonen, W.G.E.J., Kloks, C.P.A.M., Ploemen, J.-P.H.T.M., Horbach, G.J., Smit, M.J. Zandberg, P., Mellema, J.-R., Thijssen-van Zuylen, C., Tas, A.C., van Nesselrooij, J.H.J., Vogels, J.T.W.E., 2007. Sensitivity of ${ }^{1}$ H NMR analysis of rat urine in relation to toxicometabonomics. Part I: dose-dependent toxic effects of bromobenzene and paracetamol. Toxicol. Sci. 98, 271-285.

Serkova, N.J., Spratlin, J.L., Eckhardt, S.G., 2007. NMR-based metabolomics: translational application and treatment of cancer. Curr. Opin. Mol. Ther. 9, 572-585.

Siculella, L., Sabetta, S, di Summa, R., Leo, M. Giudetti, A.M., Palmieri, F., Gnoni, G.V 2002. Starvation-induced posttranscriptional control of rat liver mitochondrial citrate carrier expression. Biochem. Biophys. Res. Commun. 299, 418-423.

Sprague, C.L., Phillips, L.A., Young, K.M., Elfarra, A.A., 2004. Species and tissue differences in the toxicity of 3-butene-1, 2-diol in male Sprague-Dawley rats and B6C3F1 mice. Toxicol. Sci. 80, 3-13.

Tiziani, S., Lopes, V., Gunther, U.L., 2009. Early stage diagnosis of oral cancer using ${ }^{1} \mathrm{H}$ NMR-based metabolomics. Neoplasia 11, 269-276.

Wade, M.G., Foster, W.G., Younglai, E.V., McMahon, A., Leingartner, K., Yagminas, A. Blakey, D., Fournier, M., Desaulniers, D., Hughes, C.L., 2002. Effects of subchronic exposure to a complex mixture of persistent contaminants in male rats: systemic immune, and reproductive effects. Toxicol. Sci. 67, 131-143.

Walker, N.J., Crockett, P.W., Nyska, A., Brix, A.E., Jokinen, M.P., Sells, D.M., Hailey, J.R. Easterling, M., Haseman, J.K., Yin, M., Wyde, M.E., Bucher, J.R., Portier, C.J., 2005 Dose-additive carcinogenicity of a defined mixture of "dioxin-like compounds". Environ. Health Perspect. 113, 43-48.

Wang, Q.J., Jiang, Y., Wu, C.Q., Zhao, J.Y., Yu, S.Z., Yuan, B.L., Yan, X.Z., Liao, M.Y., 2006 Study of a novel indolin-2-ketone compound Z24 induced hepatotoxicity by NMRspectroscopy-based metabonomics of rat urine, blood plasma, and liver extracts. Toxicol. Appl. Pharmacol. 215, 71-82.

Waterfield, C.J., Turton, J.A., Scales, M.D., Timbrell, J.A., 1993. Investigations into the effects of various hepatotoxic compounds on urinary and liver taurine levels in rats. Arch. Toxicol. 67, 244-254.

Webb-Robertson, B.J., Lowry, D.F., Jarman, K.H., Harbo, S.J., Meng, Q.R., Fuciarelli, A.F., Pounds, J.G., Lee, K.M., 2005. A study of spectral integration and normalization in NMR-based metabonomic analyses. J. Pharm. Biomed. Anal. 39, 830-836.

Wu, H., Zhang, X., Li, X., Li, Z., Wu, Y., Pei, F., 2005. Comparison of metabolic profiles from serum from hepatotoxin-treated rats by nuclear-magnetic-resonance -spectroscopybased metabonomic analysis. Anal. Biochem. 340, 99-105.

Yavuz, A., Tetta, C., Ersoy, F.F., D'intini, V., Ratanarat, R., De Cal, M., Bonello, M., Bordoni, V., Salvatori, G., Andrikos, E., Yakupoglu, G., Levin, N.W., Ronco, C., 2005. Uremic toxins: a new focus on an old subject. Semin. Dial. 18, 203-211.

Yokoyama, T., Banta, S., Berthiaume, F., Nagrath, D., Tompkins, R.G., Yarmush, M.L., 2005. Evolution of intrahepatic carbon, nitrogen, and energy metabolism in a Dgalactosamine-induced rat liver failure model. Metab. Eng. 7, 88-103. 